\title{
Estimation of the walking speed of individuals with transfemoral amputation from a single prosthetic shank-mounted IMU
}

\author{
Boris Dauriac ${ }^{1,2} \oplus$, Xavier Bonnet', Helene Pillet' and Francois Lavaste'
}

\begin{abstract}
Microprocessor prosthetic knees, able to restore the gait of people with transfemoral amputation, are now often equipped with sensors embedded in the prosthetic shank, which could be used to assess some gait characteristics during real-life activities. In particular, an estimation of the walking speed during the locomotion of those subjects would be a relevant indicator of the performance. However, if methods have already been proposed in the literature to compute this walking speed, none are directly usable in this context and with this population. For these reasons, the current study proposed to estimate the instantaneous walking speed with a shank-embedded Inertial Measurement Units based on a biomechanical model of the prosthetic lower limb. Averaged walking speed estimation has been quantified for nine individuals with transfemoral amputation walking on a treadmill at different speeds and slopes when wearing an instrumented knee ankle prosthesis. Experimental results demonstrated the ability of the model to estimate the walking speed with an accuracy of $9 \%$ (normalized root mean squared errors over all the patients), which is consistent with previous reported walking speed estimation errors. In addition, as the walking speed estimation is instantaneous, the proposed method can provide the estimation by the end of the stance phase, which is an originality compared to other methods based on step length estimation. The present method is relevant for the estimation of walking speed during real-life activities of above-knee amputees opening the way to direct activity monitoring from the prosthesis.
\end{abstract}

\section{Keywords}

Walking speed, ambulatory system, gait analysis, Inertial Measurement Unit; transfemoral amputee, center of mass

\section{Introduction}

During the last two decades, microprocessor prosthetic knees, able to control the stance and the swing phases of gait, have increased the safety and quality of life of individuals with transfemoral amputation. ${ }^{1-3}$ However, to quantify prosthetic fitting objective performance requires the assessment of characteristics of the gait during real-life activities. Recently, Microelectromechanical systems technology has become affordable to develop wearable sensors that could monitor such real-life activities. Several information can be extracted from those sensors such as Inertial Measurement Units (IMU) going from segment angle estimation, ${ }^{4-7}$ gait parameters estimation ${ }^{8-10}$ to activity classification. ${ }^{11-14}$ For people with lower limb amputation, cadence, step counts and activity bout duration have been the most used as estimators of in real life conditions. ${ }^{15-21}$ On the contrary, walking speed estimation (WSE) has been rarely performed in people with amputation because of the difficulty to assess spatial parameters from wearable sensors signals. Thus, some authors have sought to link the cadence to the walking speed. ${ }^{15}$ However, cadence is not always correlated with walking speed, for instance, when walking on different inclined surfaces as the step length changes. ${ }^{22}$

Nowadays, more and more microprocessor prostheses embed IMU in the prosthetic shank, which could be used to estimate the walking speed in real life

\footnotetext{
'Arts et Metiers ParisTech, LBM/Institut de Biomecanique Humaine Georges Charpak, Paris, France

${ }^{2}$ PROTEOR, Seurre, France

Corresponding author:

Boris Dauriac, Arts et Metiers ParisTech, LBM/Institut de Biomecanique Humaine Georges Charpak, I5I bd de l'Hopital, Paris 750 I3, France.

Email: boris.dauriac@ensam.eu
} 
environment. In the literature, several methods have been developed and evaluated on different populations.

Some authors proposed to use kinematic models of the lower limbs to perform this estimation. For asymptomatic subjects, Aminian et al. proposed a stance and swing phase kinematic planar model using three gyroscopes on one thigh, and on both shanks to estimate the walking speed. Aminian et al.'s ${ }^{23}$ method was later adapted by Salarian et al. ${ }^{24}$ with only shanks instrumentation. The main drawback of this method for people with amputation is the necessity to instrument the sound limb, which jeopardizes the compliance to such protocol. Other studies have proposed estimation of walking speed for individuals with transfemoral amputation using only one IMU and a gait model. Miyazaki $^{25}$ instrumented the thigh with a gyroscope and proposed a single segment planar model of the swing phase. In the study of Lenzi et al., ${ }^{26}$ an IMU was placed on the prosthetic shank but the kinematic model used was not fully described.

Other methods have been proposed for asymptomatic subjects. For example, machine learning was used by Aminian et al. ${ }^{27}$ to estimate walking speed from a single axis accelerometer on one heel and a 3D one on the waist. If the method was proved to be accurate for one person, the generalization to other individuals is not straight forward due to overfitting and sensor placement sensitivity. ${ }^{28,29}$ Another way to quantify the walking speed consists in double integrating a segment point acceleration. Authors using this procedure, generally placed the IMU on the foot, ${ }^{30,31}$ benefiting from the stationary state of the foot during the stance phase. However, prosthesis embedded IMU are most often placed in the shank. Li et al. proposed a method of double integration to quantify the walking speed of asymptomatic people from an IMU positioned on the shank. However, this method assumes that the shank angular velocity is almost null at midstance. $^{32,33}$ This hypothesis is not adapted for people with transfemoral amputation due to the lack of knee flexion during early to midstance. ${ }^{34}$ Plus, double integration of the acceleration can also be prone to random drift computationally heavy to remove for real time application. ${ }^{35}$ Still, these methods have not been tested on this population.

In this context, the present study proposes to use a planar gait model to quantify the averaged walking speed by estimating the instantaneous velocity of the center of mass (COM) during the gait cycle using only one IMU integrated in a prosthetic shank. To evaluate this method, nine individuals with transfemoral amputation walked on a treadmill at different speeds and inclinations with a Microprocessor controlled Knee
Ankle Prosthesis (MKAP) prototype. ${ }^{36}$ Embedded sensors in the MKAP were used to compute WSE at each cycle, which was compared to the treadmill speed.

\section{Methods}

\section{Kinematic model}

The WSE method presented here is based on a kinematic planar model. It consists of an inverse pendulum model representing the prosthetic lower limb during the first half of the gait cycle (i.e. from the prosthetic heel strike to the contralateral heel strike) and considering the absence of knee flexion during this phase in the population of individuals with transfemoral amputation equipped with single axis knee prosthesis. ${ }^{34,37}$ The pelvis, the thigh, the shank and the foot are modeled by a single rigid body going from the COM to the foot-floor contact point. The prosthetic foot-ankle complex is considered to be kinematically equivalent to a rigid arc shape rolling without sliding during stance. ${ }^{38,39}$ From this model, the instantaneous COM velocity $\left(\overrightarrow{V_{C O M}}\right)$ expressed in the global frame $\left(R_{0}\right)$ with $\overrightarrow{X_{0}}$ horizontal and aligned with the floor and $\overrightarrow{Y_{0}}$ vertical and pointing upward can be inferred from only four parameters: the COM height $L$ when standing, the leg angular velocity $\dot{\theta}$ with respect to $R_{0}$, its angle $\theta$ with regard to the

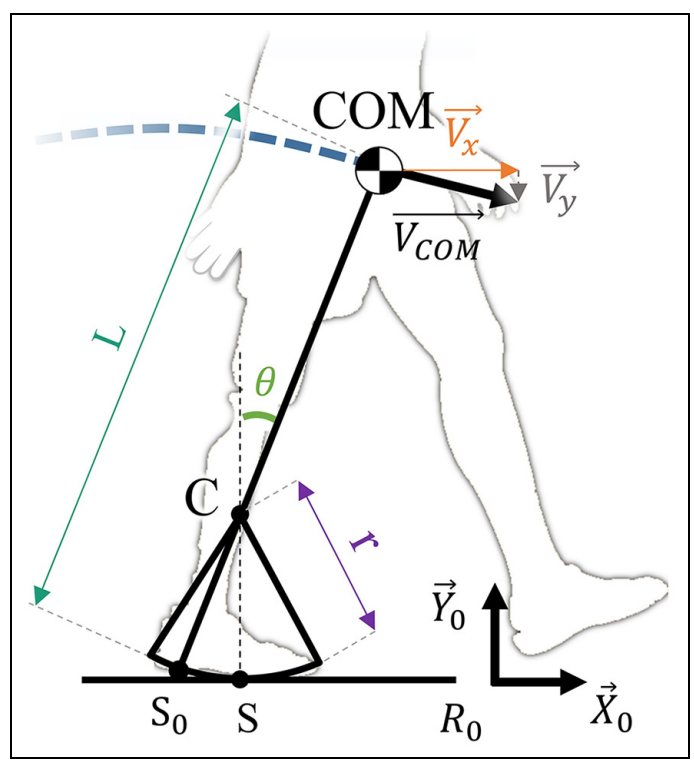

Figure I. Stance phase gait model ( $S$ contact point between the foot and the floor, $S 0$ contact point $S$ when the lower limb is vertical, $C$ : foot arc center; COM: center of mass; $L$ : COM height; $r$ : foot arc radius, $R 0$ global frame $\left(\overrightarrow{Y_{0}}\right.$ aligned with the gravity, $\overrightarrow{X_{0}}$ aligned with the walking direction), $\theta$ sagittal angle of the leg with respect to $R 0, \overrightarrow{V_{C O M}}=C O M$ velocity, $V_{x}=$ Projection of $\overrightarrow{V_{C O M}}$ on $\overrightarrow{X_{0}}, V_{y}=$ projection of $\overrightarrow{V_{C O M}}$ on $\overrightarrow{Y_{0}}$ ). 
gravity vector and the foot arc radius $r$, with $C$ its cen-

ter and $S$ the foot-floor contact point (Figure 1)

$$
\overrightarrow{V_{C O M}}=\vec{V}\left(C O M / R_{0}\right)_{R_{0}}=\vec{V}\left(C / R_{0}\right)_{R_{0}}+\vec{\Omega}\left(R_{1} / R_{0}\right)_{R_{0}} \times \overrightarrow{C C O M}_{R_{0}}=\dot{\theta}\left[\begin{array}{c}
r+(L-r) \cdot \cos (\theta) \\
-(L-r) \cdot \sin (\theta) \\
0
\end{array}\right.
$$

where $\times$ denotes the cross product. $\vec{V}\left(C O M / R_{0}\right)_{R_{0}}$ is the speed of the COM relative to the global frame $\left(R_{0}\right)$ expressed in the global frame $\left(R_{0}\right), \vec{V}\left(C / R_{0}\right)_{R_{0}}$ is the speed of the center of the foot arc expressed in $R_{0}$, $\vec{\Omega}\left(R_{1} / R_{0}\right)_{R_{0}}$ is the rotational speed of the Frame $R_{1}$ linked to the rigid body relative to $R_{0}$, and $\overrightarrow{C C O M}_{R_{0}}$ is the vector displacement from the point $C$ to $C O M$ expressed in $R_{0} \cdot \overrightarrow{V_{C O M}}$ can be decomposed into $V_{x}$ and $V_{y}$ corresponding to the $C O M$ instantaneous velocity projection along the $\overrightarrow{X_{0}}$ axis and $\overrightarrow{Y_{0}}$ axis of the global frame $R_{0}$. Moreover, $r$ and $L$ can be estimated as proportions of the body height $(\mathrm{BH})$, respectively, $19 \%,{ }^{40}$ and $58.8 \%{ }^{41}$ giving a simplified expression of the previous equation (2)

$$
\begin{aligned}
& \vec{V}\left(C O M / R_{0}\right)_{R_{0}} \cdot \overrightarrow{x_{0}}=V_{x} \\
& =\frac{B H \cdot \dot{\theta}}{100} \cdot(19+39.8 \cdot \cos (\theta))
\end{aligned}
$$

Equation (1) gives an instantaneous WSE during the stance phase on the affected side independent on the floor inclination as far as the model assumptions are verified (i.e. knee extended during stance). The average walking speed over the whole gait cycle is assumed to be equal to $V_{x}$ (equation (2)) averaged over the first half of the gait cycle. To determine the gait cycle, heel strikes were detected with an ankle moment sensor considering a threshold of $-5 \mathrm{Nm}$. $\dot{\theta}$ and $\theta$ were obtained thanks to the IMU embedded in the prosthetic shank using a complementary filter. ${ }^{6}$

\section{Experimental protocol}

Nine individuals with transfemoral amputation wearing the MKAP prototype participated in this study. Their characteristics are listed in Table 1. Different treadmill speed and slope conditions were tested by all participants depending on their capacity. All patients performed level walking at speeds ranging from $0.56 \mathrm{~m} / \mathrm{s}$ to $1.39 \mathrm{~m} / \mathrm{s}$ by $0.28 \mathrm{~m} / \mathrm{s}$ increments apart one subject who didn't perform the $1.39 \mathrm{~m} / \mathrm{s}$ condition. Three patients also walked at $1.67 \mathrm{~m} / \mathrm{s}$. Six of them also tested a $5.7^{\circ}$ slope at their self-selected speed depending on their capacity. The values of walking speed are given in Table 1. For each speed and slope condition, 7-10 cycles were recorded after the steady state was established (more than 20 steps after the treadmill speed was changed). Between 1 and $5 \mathrm{~min}$ rest was allowed between each condition. The protocol was approved by the local ethics committee (RCB 2011-A00409-32), and all participants gave their consent.

WSE has been computed for each cycle. WSE error was computed against the treadmill speed. The treadmill speed can vary depending on weight acceptance, treadmill belt deformation, and motor servo loop, this variation has been estimated to be less than $4 \%$.

\section{Data analysis}

For each treadmill walking trial, WSE was computed at each cycle. Estimation error for level and slope walking was calculated for each patient with mean error between WSE and the reference velocity $V_{\text {treadmill, }}$ standard deviation error, root mean squared error $\left(\mathrm{RMSE}=\sqrt{\sum_{n=1}^{N}\left(V_{\text {treadmill } n}-W S E_{n}\right)^{2} / N}\right.$ with $\mathrm{N}$ the number of samples) as well as normalized RMSE $\left(\mathrm{NRMSE}=\left(\sqrt{\left.\sum_{n=1}^{N}\left(V_{\text {treadmill } n}-W S E_{n}\right) / V_{\text {treadmill } n}\right)^{2} / N}\right)\right.$ were computed across all walking cycle for each slope condition. The averaged RMSE and NRMSE, mean error and standard deviation error over all patients were used to evaluate the intersubject reproducibility for each slope conditions. The agreement error was computed with the squared Pearson's correlation coefficient.

\section{Results}

Figure 2 presents WSE and WSE error against the treadmill speed considered as the reference.

The WSE method showed a good agreement with the reference speed $\left(R^{2}=0.93\right)$ for all level walking conditions. Mean NRMSE, across all patients, for level

Table I. Subjects characteristics and walking conditions conducted.

\begin{tabular}{lllll}
\hline Patient & $\begin{array}{l}\text { Height } \\
(\mathrm{m})\end{array}$ & $\begin{array}{l}\text { Mass } \\
(\mathrm{kg})\end{array}$ & $\begin{array}{l}\text { Level walking } \\
\text { speed } \\
\text { range }(\mathrm{m} / \mathrm{s})\end{array}$ & $\begin{array}{l}\text { Up slope } \\
\text { walking } \\
\text { speed }(\mathrm{m} / \mathrm{s}) \\
\text { at 5.71 }\end{array}$ \\
\hline SI & 1.86 & 73 & $0.56-1.67$ & $0.83,1.39$ \\
S2 & 1.94 & 85 & $0.56-1.67$ & 1.11 \\
S3 & 1.90 & 81 & $0.56-1.39$ & $\mathrm{n} / \mathrm{a}$ \\
S4 & 1.87 & 83 & $0.56-1.39$ & $\mathrm{n} / \mathrm{a}$ \\
S5 & 1.93 & 131 & $0.56-1.67$ & 0.83 \\
S6 & 1.59 & 61 & $0.56-1.39$ & 0.83 \\
S7 & 1.86 & 117 & $0.56-1.39$ & 0.83 \\
S8 & 1.57 & 60 & $0.56-1.11$ & $\mathrm{n} / \mathrm{a}$ \\
S9 & 1.75 & 79 & $0.56-1.39$ & 0.83 \\
\hline
\end{tabular}




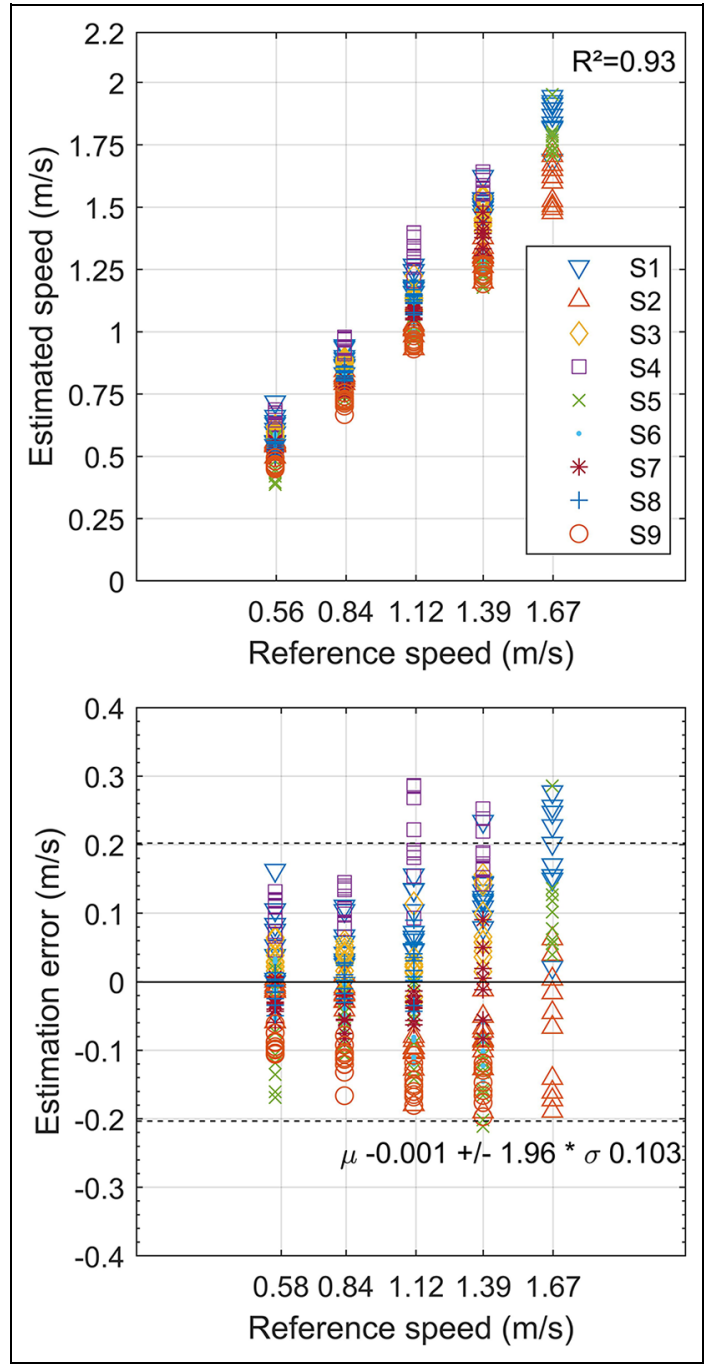

Figure 2. Top figure corresponds to WSE versus reference for each level walking speed conditions. Bottom figure corresponds to Bland-Altman plots for WSE. Each mark corresponds to a cycle WSE, each different mark corresponds to a different patient. $R^{2}$ corresponds to the overall correlation for all subjects cycles.

walking was $8.93 \%$ (Standard Deviation: $4.36 \%$; range: $3.54 \%-16.53 \%$ ). This result corresponded to a mean and standard deviation error of $-0.002 \pm 0.088 \mathrm{~m} / \mathrm{s}$. The RMSE, NRMSE, mean and standard deviation errors at level walking can be found for each subject in Table 2. For the $5.7^{\circ}$ slope condition, average NRMSE were $9.58 \%$ corresponding to $0.038 \pm 0.050 \mathrm{~m} / \mathrm{s}$ with a good agreement between the reference and the estimated walking speed $\left(R^{2}=0.93\right)$. Errors for each patient for upslope walking conditions are reported in Table 3.

\section{Discussion}

The current study presents a method to estimate the instantaneous walking speed based on a mechanical gait model using a single IMU located on the tibia and the $\mathrm{BH}$. This walking speed was averaged during the stance phase to be compared with treadmill reference speed.
Table 2. Walking speed estimation errors for each patient for level walking conditions.

\begin{tabular}{|c|c|c|c|c|}
\hline & $\begin{array}{l}\text { RMSE } \\
(\mathrm{m} / \mathrm{s})\end{array}$ & $\begin{array}{l}\text { NRMSE } \\
(\%)\end{array}$ & $\begin{array}{l}\text { Mean } \\
\text { error } \\
(\mathrm{m} / \mathrm{s})\end{array}$ & $\begin{array}{l}\text { SD } \\
\text { error } \\
(\mathrm{m} / \mathrm{s})\end{array}$ \\
\hline SI & 0.125 & 10.539 & 0.103 & 0.071 \\
\hline S2 & 0.088 & 6.820 & -0.063 & 0.062 \\
\hline S3 & 0.059 & 5.918 & 0.043 & 0.041 \\
\hline S4 & 0.163 & 16.528 & 0.152 & 0.061 \\
\hline S5 & 0.109 & 11.234 & -0.047 & 0.099 \\
\hline S6 & $0.07 \mathrm{I}$ & 6.663 & -0.056 & 0.044 \\
\hline S7 & 0.046 & 5.178 & -0.029 & 0.036 \\
\hline S8 & 0.031 & 3.537 & 0.003 & 0.031 \\
\hline S9 & 0.130 & 13.913 & -0.126 & 0.033 \\
\hline Mean & 0.091 & 8.926 & -0.002 & 0.053 \\
\hline Standard deviation & 0.044 & 4.363 & 0.088 & 0.022 \\
\hline
\end{tabular}

RMSE: root mean squared error; NRMSE: normalized root mean squared error.

Table 3. Walking speed estimation errors for each patient for upslope $\left(+5.7^{\circ}\right)$ walking conditions.

\begin{tabular}{lrrrr}
\hline & $\begin{array}{l}\text { RMSE } \\
(\mathrm{m} / \mathrm{s})\end{array}$ & \multicolumn{1}{l}{$\begin{array}{l}\text { NRMSE } \\
(\%)\end{array}$} & $\begin{array}{l}\text { Mean } \\
\text { error } \\
(\mathrm{m} / \mathrm{s})\end{array}$ & \begin{tabular}{l}
\multicolumn{1}{l}{ SD } \\
error \\
$(\mathrm{m} / \mathrm{s})$
\end{tabular} \\
\hline S1 & 0.118 & 11.091 & 0.110 & 0.044 \\
S2 & 0.104 & 9.382 & 0.097 & 0.042 \\
S5 & 0.161 & 19.378 & 0.124 & 0.108 \\
S6 & 0.029 & 3.486 & -0.027 & 0.012 \\
S7 & 0.029 & 3.527 & -0.023 & 0.020 \\
S9 & 0.089 & 10.635 & -0.053 & 0.075 \\
\hline Mean & 0.088 & 9.583 & 0.038 & 0.050 \\
Standard deviation & 0.052 & 5.886 & 0.080 & 0.036 \\
\hline
\end{tabular}

RMSE: root mean squared error; NRMSE: normalized root mean squared error.

The WSE error, obtained for individuals with transfemoral amputation, was inferior to $16.53 \%$ during level walking, which is consistent with the results of Lenzi et al. ${ }^{26}$ ( $8 \%$ error, number of subjects not reported) and Miyazaki $^{25}$ (error inferior to $15 \%$, number of subjects $=7$ ). Mean WSE error is very close to the one obtained by Aminian et al. ${ }^{23}$ with both lower limbs instrumented for twenty asymptomatic subjects or his modified single limb instrumentation method for ten asymptomatic subjects $^{24}(0.06 \mathrm{~m} / \mathrm{s}$ error for both models). For their machine learning approach, Aminian et al. ${ }^{27}$ only reported the maximum coefficient of variation on the distance estimated, making it difficult to compare the proposed method with theirs. Finally, the presented results are in the range of the double integration methods presented by Sabatini et al. ${ }^{31}(0.05 \mathrm{~m} / \mathrm{s}$ error) and Yang et al. ${ }^{33}$ (4.2\% error). Table 4 hereafter compares the WSE error reported in the literature according to the method used, the number of subjects and the population considered and the sensors and their placement. 
Table 4. Walking speed estimation reported in the literature.

\begin{tabular}{|c|c|c|c|c|c|}
\hline Author & $N$ subject & Population & Sensors & Method & Results \\
\hline Miyazaki ${ }^{25}$ & 7 & TF & Thigh gyroscope & Kinematic model & Inferior to $15 \%$ relative error \\
\hline Lenzi et al. ${ }^{26}$ & ? & $\mathrm{TF}$ & Shank IMU & Double integration & $8 \%$ \\
\hline Aminian et al. ${ }^{27}$ & 6 & SA & Foot and waist IMU & Machine learning & $\begin{array}{l}\text { Maximum coefficient of } \\
\text { variation } 6 \% \text { on the total } \\
\text { distance covered }\end{array}$ \\
\hline Aminian et al. ${ }^{23}$ & 20 & SA & $\begin{array}{l}\text { Shank and thigh gyroscope } \\
\text { on both legs }\end{array}$ & Kinematic model & RMSE 0.06 m/s (6.7\%) \\
\hline Sabatini et al. ${ }^{31}$ & 5 & SA & Foot IMU & Double integration & RMSE $0.05 \mathrm{~m} / \mathrm{s}$ \\
\hline Yang et al. $^{33}$ & 16 & SA & Shank IMU & Double integration & RMSE $4.2 \%$ \\
\hline Salarian et al. ${ }^{24}$ & 10 & SA & $\begin{array}{l}\text { Shank gyroscope } \\
\text { on both legs }\end{array}$ & Kinematic model & $0.04 \pm 0.06 \mathrm{~m} / \mathrm{s}$ \\
\hline This study & $\underline{9}$ & $\underline{\text { TF }}$ & $\underline{\text { Shank IMU }}$ & $\underline{\text { Kinematic model }}$ & $\frac{\text { RMSE } 0.09 \mathrm{~m} / \mathrm{s}(9 \%)}{\text { on level walking }}$ \\
\hline
\end{tabular}

TF: individuals with transfemoral amputation; SA: individuals without amputation; IMU: Inertial Measurement Units; RMSE: root mean squared error

As concerns the instrumentation of the prosthesis, to our knowledge, previous methods, available in the literature and developed for asymptomatic subjects, almost all required sensors on other parts of the body or on the foot. ${ }^{23,24,30}$ The foot placement of the sensor jeopardizes the use of a generic instrumented prosthetic component as the prosthetic foot must be adapted to each patient shoe size. Only Yang et al. proposed a method to compute WSE from a shank embedded IMU but it was not adapted for this population. ${ }^{32,33}$ On the contrary, the model proposed in the present study was specifically designed for individuals with transfemoral amputation and revealed relevant for this specific population given the reported values of the estimation error.

An additional advantage of the method is to give an instantaneous WSE during the stance phase using only one IMU embedded in the prosthetic shank. In the literature, the estimation of the walking speed generally relied on the estimation of the step length, or foot displacement which could be obtained at the end of the gait cycle. Therefore, prosthetic behavior changes, based on this evaluation, cannot be effective before the next cycle. ${ }^{23,33}$ On the contrary, the instantaneous WSE could be averaged at the end of the stance phase but could also be used to change the prosthesis behavior as soon as the swing phase starts.

Compared to the treadmill reference speed, the method underestimates the averaged walking speed. The uncertainty of the estimation of the COM height from the anthropomorphic model ${ }^{41}$ directly affects the estimation of the COM velocity and could be evaluated to about $\pm 1 \%$ of the $\mathrm{BH}^{42}$ In addition, as the kinematic model is planar, the pelvis transversal movements that have an impact on the COM position in the sagittal plane are neglected. Also, the method assumes the equality of the average COM speed on both half portions of the cycle. Asymmetry induced by gait deviations such as hip hiking, vaulting or pelvis transverse rotation ${ }^{43}$ could also have an impact on this hypothesis. Results also showed that the estimation error was affected when walking in slope. The WSE error increased up to $9.58 \%$ for the mean NRMSE. A part of this error can be attributed to the forward velocity projection on the horizontal plane $(<1 \%$ in this study slope condition). Another part of this error could be due to the increase of the asymmetry in this situation. ${ }^{44}$

Finally, the WSE has been performed on a treadmill. Gait on treadmill is known to differ from overground walking. ${ }^{45}$ However, the performance of the method should not be deteriorated overground as the assumptions made would not be disputed. Thus, even if the gait on treadmill is not completely representative of overground walking, we could expect similar results in the latest condition as the ones reported here.

To conclude, this study presents a method to estimate the walking speed for individuals with transfemoral amputation using a single shank embedded IMU. A mean 9\% RMSE errors have been quantified with nine patients walking on a treadmill at a $0^{\circ}$ slope. In a 5\% slope, the RMSE errors slightly increased but remained acceptable for an estimation of the patient activity. The validation of the method should be completed by additional acquisitions in a real environment to show its relevance for the estimation of walking speed during real-life activities of above-knee amputees.

\section{Declaration of conflicting interests}

The author(s) declared the following potential conflicts of interest with respect to the research, authorship, and/ or publication of this article: The corresponding author Boris Dauriac was an employee of Proteor, the company which developed the prosthesis used in this study. The other authors declare that there is no conflict of interest.

\section{Funding}

The author(s) disclosed receipt of the following financial support for the research, authorship, and/or publication of this article: The corresponding author Boris Dauriac was funded by a CIFRE grant from the Proteor company, subsidized by the French National Association for Research and Technology (ANRT, 
CIFRE grant 2014/1047). The other authors received no financial support for the research, authorship, and/ or publication of this article.

\section{ORCID iD} Boris Dauriac (iD https://orcid.org/0000-0003-1804-
$862 \mathrm{X}$

\section{References}

1. Sawers AB and Hafner BJ. Outcomes associated with the use of microprocessor-controlled prosthetic knees among individuals with unilateral transfemoral limb loss: a systematic review. J Rehabil Res Dev 2013; 50: 273.

2. Schmalz T, Blumentritt S and Marx B. Biomechanical analysis of stair ambulation in lower limb amputees. Gait Posture 2007; 25: 267-278.

3. Burnfield JM, Eberly VJ, Gronely JK, et al. Impact of stance phase microprocessor-controlled knee prosthesis on ramp negotiation and community walking function in K2 level transfemoral amputees. Prosthet Orthot Int 2012; 36: 95-104.

4. Sabatini AM. Estimating three-dimensional orientation of human body parts by inertial/magnetic sensing. Sensors 2011; 11: 1489-1525.

5. Sabatini AM. Kalman-filter-based orientation determination using inertial/magnetic sensors: observability analysis and performance evaluation. Sensors 2011; 11: 9182 9206.

6. Mahony R, Hamel T and Pflimlin JM. Nonlinear complementary filters on the special orthogonal group. IEEE T Automat Contr 2008; 53: 1203-1218.

7. Seel T, Raisch $\mathrm{J}$ and Schauer T. IMU-based joint angle measurement for gait analysis. Sensors 2014; 14: 68916909.

8. Tura A, Raggi M, Rocchi L, et al. Gait symmetry and regularity in transfemoral amputees assessed by trunk accelerations. J Neuroeng Rehabil 2010; 7: 4.

9. Mariani B, Rochat S, Büla CJ, et al. Heel and toe clearance estimation for gait analysis using wireless inertial sensors. IEEE T Biomed Eng 2012; 59: 3162-3168.

10. Dadashi F, Mariani B, Rochat S, et al. Gait and foot clearance parameters obtained using shoe-worn inertial sensors in a large-population sample of older adults. Sensors 2013; 14: 443-457.

11. Yuan K, Wang Q and Wang L. Fuzzy-logic-based terrain identification with multisensor fusion for transtibial amputees. IEEE/ASME T Mech 2015; 20: 618-630.

12. Redfield MT, Cagle JC, Hafner BJ, et al. Classifying prosthetic use via accelerometry in persons with transtibial amputations. $J$ Rehabil Res Dev 2013; 50: 1201-1212.

13. Zhang F, Fang Z, Liu M, et al. Preliminary design of a terrain recognition system. Conf Proc IEEE Eng Med Biol Soc 2011; 2011: 5452-5455.

14. Sprager S and Juric MB. Inertial sensor-based gait recognition: a review. Sensors 2015; 15: 22089-22127.

15. Ramstrand $\mathrm{N}$ and Nilsson K-A. Validation of a patient activity monitor to quantify ambulatory activity in an amputee population. Prosthet Orthot Int 2007; 31: 157166.
16. Bussmann HB, Reuvekamp PJ, Veltink PH, et al. Validity and reliability of measurements obtained with an "activity monitor" in people with and without a transtibial amputation. Phys Ther 1998; 78: 989-998.

17. Dudek NL, Khan OD, Lemaire ED, et al. Ambulation monitoring of transtibial amputation subjects with patient activity monitor versus pedometer. $J$ Rehabil Res Dev 2008; 45: 577-585.

18. Halsne EG, Waddingham MG and Hafner BJ. Longterm activity in and among persons with transfemoral amputation. $J$ Rehabil Res Dev 2013; 50: 515.

19. Jayaraman A, Deeny S, Eisenberg Y, et al. Global position sensing and step activity as outcome measures of community mobility and social interaction for an individual with a transfemoral amputation due to dysvascular disease. Phys Ther 2014; 94: 401-410.

20. Albert MV, Deeny S, McCarthy C, et al. Monitoring daily function in persons with transfemoral amputations using a commercial activity monitor: a feasibility study. $P M \& R$ 2014; 6: 1120-1127.

21. Albert MV, McCarthy C, Valentin J, et al. Monitoring functional capability of individuals with lower limb amputations using mobile phones. PLoS ONE 2013; 8: e65340.

22. Sun J, Walters M, Svensson N, et al. The influence of surface slope on human gait characteristics: a study of urban pedestrians walking on an inclined surface. Ergonomics 1996; 39: 677-692.

23. Aminian K, Najafi B, Büla $C$, et al. Spatio-temporal parameters of gait measured by an ambulatory system using miniature gyroscopes. J Biomech 2002; 35: 689699.

24. Salarian A, Burkhard PR, Vingerhoets FJG, et al. A novel approach to reducing number of sensing units for wearable gait analysis systems. IEEE T Biomed Eng 2013; 60: $72-77$.

25. Miyazaki S. Long-term unrestrained measurement of stride length and walking velocity utilizing a piezoelectric gyroscope. IEEE T Biomed Eng 1997; 44: 753-759.

26. Lenzi T, Hargrove L and Sensinger J. Speed-adaptation mechanism: robotic prostheses can actively regulate joint torque. IEEE Robot Autom Mag 2014; 21: 94-107.

27. Aminian K, Robert $P$, Jéquier E, et al. Incline, speed, and distance assessment during unconstrained walking. Med Sci Sport Exer 1995; 27: 226-234.

28. Duda OR, Hart EP, Stork GD, et al. Pattern classification. 2nd Revised. Berlin: Springer, 2000.

29. Sabatini AM. Inertial sensing in biomechanics. In: Palaniswami $\mathrm{M}$ and Begg $\mathrm{R}$ (eds) Computational intelligence for movement sciences neural networks and other emerging techniques. London: Idea Group Publishing, pp.70-100.

30. Mannini A and Sabatini AM. Walking speed estimation using foot-mounted inertial sensors: comparing machine learning and strap-down integration methods. Med Eng Phys 2014; 36: 1312-1321.

31. Sabatini AM, Martelloni C, Scapellato S, et al. Assessment of walking features from foot inertial sensing. IEEE T Biomed Eng 2005; 52: 486-494.

32. Li Q, Young M, Naing V, et al. Walking speed estimation using a shank-mounted inertial measurement unit. J Biomech 2010; 43: 1640-1643. 
33. Yang S, Laudanski A and Li Q. Inertial sensors in estimating walking speed and inclination: an evaluation of sensor error models. Med Biol Eng Comput 2012; 50: 383-393.

34. Segal AD, Orendurff MS, Klute GK, et al. Kinematic and kinetic comparisons of transfemoral amputee gait using C-Leg and Mauch SNS prosthetic knees. $J$ Rehabil Res Dev 2006; 43: 857-870.

35. Martin E, Shia V and Bajcsy R. Determination of a patient's speed and stride length minimizing hardware requirements. In: Proceedings of the international conference on body sensor networks, Dallas, TX, 23-25 May 2011, pp.144-149. New York: IEEE.

36. Bonnet X, Djian F, Drevelle X, et al. Design and preliminary evaluation of a microprocessor controlled ankle-knee prosthetic system for above knee amputees. In: Proceedings of the 13th international symposium on $3 D$ analysis of human movement, Lausanne, 14-17 July 2014.

37. Bellmann M, Schmalz T, Ludwigs E, et al. Immediate effects of a new microprocessor-controlled prosthetic knee joint: a comparative biomechanical evaluation. Arch Phys Med Rehabil 2012; 93: 541-549.

38. Hansen AH, Childress DS, Miff SC, et al. The human ankle during walking: implications for design of biomimetic ankle prostheses. J Biomech 2004; 37: 14671474.

39. McGeer T. Passive dynamic walking. Int J Rob Res 1990; 9: $62-82$.

40. Hansen AH and Childress DS. Effects of shoe heel height on biologic rollover characteristics during walking. $J$ Rehabil Res Dev 2004; 41: 547-554.

41. Clauser CE, McConville JT, Young JW, et al. Weight, volume and center of mass of segments of the human body. AMRL technical report, Wright Patterson Air Force Base, May 1969.

42. Virmavirta $M$ and Isolehto J. Determining the location of the body's center of mass for different groups of physically active people. J Biomech 2014; 47: 1909-1913.

43. Gailey R, Allen K, Castles J, et al. Review of secondary physical conditions associated with lower-limb amputation and long-term prosthesis use. $J$ Rehabil Res Dev 2008; 45: 15-29.

44. Schaarschmidt M, Lipfert SW, Meier-Gratz C, et al. Functional gait asymmetry of unilateral transfemoral amputees. Hum Mov Sci 2012; 31: 907-917.

45. Riley PO, Paolini G, Della Croce U, et al. A kinematic and kinetic comparison of overground and treadmill walking in healthy subjects. Gait Posture 2007; 26: 17-24. 